\title{
26 Research Square \\ De-Novo Malignancies After Liver Transplantation: Experience of A High-Volume Center
}

\author{
Tevfik Tolga Sahin \\ Inonu Universitesi Tip fakultesi

\section{Zeki Ogut} \\ Inonu university \\ Volkan Ince \\ Inonu university \\ Sertac Usta \\ Inonu university \\ Sezai Yilmaz \\ Inonu University
}

Kutay Saglam ( $\nabla$ saglamk@gmail.com )

Inonu Universitesi Tip fakultesi https://orcid.org/0000-0002-0919-8370

\section{Research Article}

Keywords: de-novo, hepatic, immunosuppression, cancer

Posted Date: October 28th, 2021

DOI: https://doi.org/10.21203/rs.3.rs-1012805/v1

License: (c) (i) This work is licensed under a Creative Commons Attribution 4.0 International License. Read Full License

Version of Record: A version of this preprint was published at Journal of Gastrointestinal Cancer on November 14th, 2021. See the published version at https://doi.org/10.1007/s12029-021-00749-0. 


\section{Abstract}

\section{Purpose}

Patient care, newer immunosuppressive medications, and advances in surgical technique, have resulted in significant prolongation of survival after liver transplantation in recent years. However, as life expectancy increased and the early mortality rates have decreased, different problems have evolved due to chronic immunosuppressive therapy. The aim of the present study is to evaluate patients who were transplanted and then developed de novo malignancies, in terms of the type of malignancies and the follow-up period.

\section{Methods}

The study was conducted on 2814 patients who received liver transplantation between 2008 to 2020 in Inonu University Liver Transplant Institute. In total, the data of 23 patients were evaluated retrospectively.

\section{Results}

Non-melanoma skin cancer was the most common de-novo malignancy $(21.7 \%)$, followed by gynecological cancers (17.3\%). The interval between the time of transplantation until the development of de novo malignancy was 36 (6-75) months. The median follow-up period after the diagnoses of the de novo malignancies was 4.11 years. One, 3, 5-year survival rates of patients after the diagnoses of de novo malignancies were $69.6 \%, 56.5 \%$ and $41.9 \%$; respectively.

\section{Conclusion}

Non-melanotic skin cancers were the most common de novo cancers in liver transplant recipients. A strict surveillance programs is very important in the follow-up of liver transplant recipients.

\section{Introduction}

Liver transplantation (LT) is the gold standard treatment for end stage liver disease. In last two decades, due to the advancements made in surgical technique, immunosuppressive drugs, and the postoperative intensive care, the long-term survival rates following LT have reached $85-90 \%$. However, as life expectancy increased and the early mortality rates decreased, different problems have evolved in association with chronic immunosuppressive therapy. De-novo malignancies are one of the leading long term causes of mortality in transplant recipients [1]. The incidence ranges between $2.6 \%$ to $33.6 \%$, which is 2 to 4 times more than normal the population [2,3]. The incidence types in de-novo malignancies differ according to the geographic location, primary liver disease, immunosuppressive medication, age of the patient, number of rejection episodes, alcohol and tobacco consumption of the individual [4]. One of the main reason for the development of de novo malignancies is the immunosuppressive medication that is used to prevent rejection episodes [2]. The mechanisms of action of the immunosuppressive medications, dose and duration of the treatment has paramount importance. 
Our literature search showed that there are various studies regarding risk factors associated with de novo malignancies, including the role of the type of immunosuppressive therapy which has significance for patient follow-up in these circumstances [3].

The aim of the present study is to evaluate patients with de novo malignancies who were transplanted, in terms of the types of malignancies, the follow-up period, and the surveillance protocol that is needed to be performed for these patients. We also aimed to investigate the impact of de-novo malignancies on the survival of the patients.

\section{Patients And Methods}

\section{Study Design}

The study was conducted on patients who received liver transplantation between 2008 to 2020 in Inonu University Liver Transplant Institute. In total, 2814 patients were transplanted during the study period. The electronic patient database was retrospectively searched for the recipients who were diagnosed and treated for cancer in the post-transplant period. The database search was conducted by searching specific international classification of disease-10 codes (ICD-10 codes) on the electronic database of the hospital. Recipients who were pathologically confirmed to have cancer in the post-transplant period were included in our study. Patients with preoperative diagnosis of hepatocellular cancer, neuroendocrine metastasis, other malignancies that are in remission who had postoperative recurrence of their disease were excluded from the study. Furthermore, patients who had incidental hepatocellular cancer (HCC) in the explant pathology were also excluded from analysis in our study. In total there were 23 patients who met the inclusion criteria of the present study. The study was approved by the institutional review board of Inonu University (Approval No: 2021/1643). Follow-up protocol of patients after liver transplantation

In our country, living donor liver transplantation can be performed from first to fourth degree relatives of the recipient and our institute strictly adheres to this principle. In the postoperative early period, a Doppler ultrasound examination is performed twice daily for the 5 first postoperative days. In the following 5 days, Doppler examination is done once a day. In suspicious cases, multidetector computerized tomography is performed. However, multidetector computerized tomography is performed routinely after the postoperative 10th day. We usually use low molecular weight heparin until the postoperative first week and continue with acetyl salicylic acid. Patients receive $500 \mathrm{mg}$ methyl prednisolone intraoperatively after the arterial anastomosis and it is continued in the postoperative period. The dose is gradually tapered, and it is discontinued in the postoperative $3-6^{\text {th }}$ month. Tacrolimus is started on the postoperative third day and dose is increased until the trough levels of $7-10 \mathrm{ng} / \mathrm{mL}$ are obtained. It is gradually tapered after the first month of the operation and usually kept around $4-7 \mathrm{ng} / \mathrm{mL}$. Mycophenolate mofetil is started at the same period as tacrolimus, for patients 12 years or older and continued until postoperative $6^{\text {th }}$ month. If a patient has abnormal renal functions, tacrolimus is tapered or discontinued and everolimus is stated and trough levels of 5 to $8 \mathrm{ng} / \mathrm{mL}$ is anticipated. 
The recipients are requested to reside in the city of Malatya where our institute is situated, for the pertransplant period and are called to the outpatient clinic weekly for the first postoperative three months. Thereafter, patients are checked every two weeks. Between postoperative 6 and 12 months, patients are called for monthly visits. After the first post-transplant year, outpatient clinics are requested once every 2 to 3 months. Our routine evaluation tests include complete blood count, biochemical analysis, tumor markers, serologic analysis for viral antigens and trough levels of the immunosuppressives. Routine abdominal ultrasound and chest $\mathrm{x}$ ray is done monthly. T-tube cholangiography is obtained once every 2 months and just before the T-tube will be drawn out which is usually around postoperative $6^{\text {th }}$ month. Computerized tomography is usually obtained twice every year. Patients are evaluated according to symptoms. If the patient has any gastrointestinal symptom such as epigastric pain, bloating, changes in defecation habits etc., then upper and lower gastrointestinal system endoscopy are performed. Patients are evaluated by our dermatology department in case of any cutaneous manifestations.

\section{Study Parameters}

Demographic characteristics, etiology of liver failure, operative parameters such as donor age and relation with the recipient immunosuppressive medications, type of de novo malignancy, interval between the transplant and the diagnosis of the malignancies, therapies for de novo malignancies and overall survival and survival periods after the diagnosis of de novo malignancies were all evaluated retrospectively for every patient.

\section{Statistical Analysis}

The continuous variables were expressed as median and range (Min-Max). Categorical variables are expressed as number of affected individuals and percentage of the study population ( $n$;\%). Survival analyses were performed using Kaplan-Meier Estimates. All statistical analyses were performed on Statistical Package for Social Sciences Software version 26 (SPSSv26, USA).

\section{Results}

\section{General Demographic, Clinical and Operative Characteristics of The Patients}

In total, 23 patients had de novo malignancy in the present study. The median age of the patients was 59 (1-66)years. Most of the indications for liver transplantation was hepatitis B virus [HBV] related cirrhosis [n=12;52.2\%]. Twenty patients received a living donor liver graft and 3 patients [13\%] received deceased donor liver graft. In 18 recipients (90\%), the donor was first degree relative. Median age of the living donors were 28 (20-45) years and for the deceased donors it was 75.5 (20-78) years. Table-1 summarizes the demographic and clinical parameters of the patients included in the study.

\section{Characteristics of The De-Novo Malignancies}


The type of de novo malignancies varied widely. Non-melanoma skin cancer developed in 5 patients (21.7\%), gynecological cancers developed in 4 patients (17.3\%), post-transplant lymphoproliferative disorders (PTLD) developed in 2 patients (8.7\%), myeloproliferative disorder in 2 patients (8.7\%), colon cancer in 2 patients (8.7\%) and non-Hodgkin lymphoma in 1 patient $(4.3 \%)$. Other rare malignancies that were observed in our cohort were malignant melanoma $(n=1)$, small bowel neuroendocrine tumor $(n=1)$, renal cell carcinoma $(n=1)$, parotid carcinoma $(n=1)$, nasopharyngeal carcinoma $(n=1)$, lung cancer $(n=1)$, and Kaposi's sarcoma $(n=1)[5]$.

The median interval between the time of transplantation until the development of de novo malignancy was 36 (6-75) months. Solid organ tumors developed at a median of 31 (5-101) months and lymphoproliferative disorders developed at a median of 38.5 (13-40) months. Six patients had distant metastasis at the time of diagnosis and 5 of them died from their tumor. Therefore, the most frequent de novo malignancy observed in the present study was non-melanoma skin cancer.

\section{Characteristics of Patients with Non-Melanoma Skin Cancer}

In total, 5 patients had non-melanoma skin cancer. 3 were male and 2 were female. The median interval between the transplant procedure and the diagnosis of skin cancer was 37 (6-75) months. The median age of the patients with non-melanoma skin cancers were 59 (36-66) years. There was only one pediatric patient. In this patient a squamous cell carcinoma developed at the wound edge. The closure of the incision was performed by Bogota bag. In the remaining 4 patients 2 had squamous cell carcinoma and the remaining two patients had basal cell carcinoma. Only one patient in this group died 3 months after the diagnosis, the cause of death was very aggressive cancer [6] (Figure 1).

\section{Characteristics of Patients with Malignancies of the Reproductive System}

Malignancies of the reproductive system developed in 4 patients. Three of these patients had breast cancer and the remaining one patient had ovarian cancer. Median age of the patients were 52 (36-60) years. All patients were female. Median interval between the transplant procedure and the diagnosis of cancer was 31 (6-75) months. Breast cancer developed at median of 55 (5-75) months following LT. One patient with breast cancer had distant metastasis at initial diagnosis. Only in two patients with breast cancer, the disease was diagnosed at $5^{\text {th }}$ and $8^{\text {th }}$ months following the transplant procedure. None of the patients in this group dies due to cancer.

\section{Characteristics of Patients with Lymphoproliferative Disorders}

PTLD developed in 2 patients. Both were male and their ages were 49 and 52 years. The indications for liver transplantation were alcoholic cirrhosis $(n=1)$ and HBV related $\mathrm{HCC}(\mathrm{n}=1)$. One patient developed $B$ cell lymphoma of the lungs and the other patient developed T cell lymphoma. B cell lymphoma of the lungs were diagnosed at post-transplant 37 months. The patient T cell lymphoma died 2.5 months after the diagnosis and patient with B cell lymphoma of the lungs died 41.2 months after the initial diagnosis. Both patients succumbed to their malignancies. 


\section{Characteristics of 2 Patients with Myeloproliferative Disorders and Non-Hodgkin Lymphoma}

The patient with non-Hodgkin lymphoma was 61 years old male patient who received liver transplantation for HBV related cirrhosis and HCC. The disease was diagnosed 40 months after the transplant procedure and the patient is still alive.

One of the patients with lymphoproliferative disorders was a 1-year-old girl who received liver transplantation for progressive familial intrahepatic cholestasis (PFIC). She was diagnosed with acute lymphoblastic leukemia 24 months after the transplant procedure. Her polymerase chain reaction (PCR) was positive for both cytomegalovirus (CMV) and Epstein-Barr Virus (EBV). She is still in remission 63 months after the diagnosis, after receiving appropriate treatment. The other patient with myeloproliferative disorder was a 59-year-old male patient who was transplanted for cryptogenic cirrhosis. He developed multiple myeloma 13 months after the transplant procedure and died 12.8 months after the diagnosis.

\section{The Impact of De-Novo Malignancies on Post-Transplant Prognosis and Survival.}

In the present study, 10 patients (43.5\%) are still alive after the diagnosis of de novo malignancy. Furthermore, $6(26 \%)$ of the patients had metastatic disease and 5 have died due their malignancies. The median post-transplant follow-up of the patients were 7.75 years and median follow-up period after the diagnoses of the de novo malignancies was 4.11 years. The overall, 1-, 3- and 5-year survival rate of the total cohort of patients after transplantation were $91.3 \%, 78.3 \%$ and $62.9 \%$; respectively. Whereas $1-$, 3and 5-year survival rate of the patients after the diagnosis of de novo malignancies were $69.6 \%, 56.5 \%$ and $41.9 \%$; respectively (Figure 2 ).

The meansurvival duration of the patients with different types of de novo malignancies and the 1-, 3- and 5-year survival rates of the patients with different de-novo malignancies are summarized in Figure $3 \mathrm{~A}$ and B. The difference that is observed between different malignancies did not reach statistical significance; however best prognosis and survival periods were observed in patients with non-melanoma skin cancer, whereas poor prognosis and survival intervals were observed in patients with hematologic malignancies.

\section{Discussion}

De-novo malignancies are a common cause of long-term mortality [3]. De novo malignancies are responsible for approximately $20 \%$ of deaths after liver transplantation.[1]. The immunosuppressive state reduces the immune surveillance mechanisms against malignant transformation and increases susceptibility to infection with oncogenic viruses. In addition, immunosuppressive agents such as antimetabolites reduce the organism's capability of deoxyribonucleotide acid (DNA) repair which results in accumulating DNA damage that can lead to carcinogenesis[1].

The results of the present study suggested that common de novo malignancies were non-melanoma skin cancer; followed by gynecologic malignancies and PTLD. The type of cancers shows geographic 
variability. Furthermore, gender, race and age affect the incidence and the type of cancer that is seen in post-transplant period. This is a main reason for the discrepancy between the Asian and Western literature. In Japan, the most common cancer is reported to be gastric cancer, in Korea it is reported to be colon cancer and in Western countries, non-melanotic skin cancer and PTLD were reported as the most common de-novo malignancies in the post-transplant period [3,7-9].

Skin cancer is the most common de-novo malignancy observed in solid organ transplant recipients. The incidence of squamous cell carcinoma increases 65 -fold in solid organ transplant recipients when compared to the normal population. Similarly, the incidence of malignant melanoma increases 3-fold in solid organ transplant recipients when compared to the normal population [10]. The risk factors for nonmelanoma skin cancers in liver transplant recipients include male gender, older age, skin type, and total sun exposure burden [11]. In the present study, $60 \%$ of the patients were male and in one patient, skin cancer developed due to chronic inflammation.

PTLD is the most common de-novo malignancy that affects transplant recipients. It is a spectrum of diseases seen in solid organ and tissue recipients that range from benign lymphoid hyperplasia to malignant diseases such lymphoma. It has different subtypes that include early lesions, polymorphic PTLD, monomorphic PTLD (diffuse B cell and T cell lymphoma), and classic non-Hodgkin lymphoma [12]. Almost $90 \%$ of the cases are associated with EBV [12]. There were 2 patients with PTLD (B-cell lymphoma $(n=1)$ and T-cell lymphoma $(n=1))$ in this study and both had a poor prognosis that resulted in death.

Malignancies of the female reproductive system were the second most common de-novo malignancy observed in liver transplant recipients in our study. Most of the patients had breast cancer and cancer and were diagnosed at a median of 55 months following LT. Only one patient was diagnosed with distant metastasis at diagnosis. Studies suggest that the relative risk of breast cancer is not increased following solid organ transplantation [9]. However, our results showed that breast cancer is among the common de novo malignancies in liver transplant recipients. Estrogenic stimulation may results in malignant transformation in patients who have risk factors [13]. For this reason, regular surveillance for breast cancer is warranted in these patients both in the pre- and post-transplant period.

The median age of the patients in the present study were 59 (1-66) years and there was a male predominance. Kaneko et al have reported that younger patients and females had a higher risk of developing de-novo malignancies [7]. Bhat et al emphasized that transplant age was a significant risk factor for de novo cancer development [14]. Manzia et al have reported that de novo cancer risk increases by 19 folds in pediatric patients, and they have shown that aggressive and treatment resistant tumors have developed [3].

In the present study, solid organ tumors developed at a median of 31 (5-101) months and lymphoproliferative disorders developed at a median of 38.5 (13-40) months. Various studies have reported a 5- 6-year interval between the transplant procedure and diagnosis of de-novo cancer $[1,15]$. 
Our results found that de novo cancers can develop earlier than in those reports. This may be a geographic difference and emphasizes the importance of strict surveillance programs.

Studies have reported that more advanced cancers were diagnosed in solid organ transplant recipients and solid organ tumors such as lung and colon cancer and other malignancies such as PTLD were major causes of mortality in liver transplant recipients $[1,16]$. In the present study, patients with non-melanoma skin cancer showed the best overall survival after diagnosis when compared to other solid organ tumors and PTLD. Furthermore, 6 patients had distant metastasis at the time of diagnosis and mortality was observed in 5 of them (83.3\%).

\section{Conclusion}

In summary, non-melanotic skin cancers were the most common de novo cancers in liver transplant recipients. Fortunately, the prognosis of these patients is very good. Another important finding of the present study is that cancers of the reproductive system were the second most common de-novo cancers that developed in these patients. These two common de-novo cancer types suggest that strict surveillance programs are very important in the follow-up of liver transplant recipients. The de novo cancers in the present study developed within 3 years after the transplant procedure. The prognosis was worse in patients with lymphoproliferative disorders and hematologic malignancies and in colorectal cancer.

\section{Abbreviations}

LT: Liver transplantation

ICD-10 : international classification of disease 10

HCC: hepatocellular cancer

HBV: hepatitis B virus

PLTD: post-transplant lymphoproliferative disorders

PFIC: progressive familial intrahepatic cholestasis

PCR: polymerase chain reaction

CMV: cytomegalovirus

EBV: Epstein-Barr Virus

DNA: deoxyribonucleotide acid

\section{Declarations}




\section{Compliance With Ethical Standarts}

Funding: No funding was received for conducting this study.

Conflict of interest: The authors declare that they have no conflict of interest.

\section{References}

1. Sérée O, Altieri M, Guillaume E, De Mil R, Lobbedez T, Robinson P, et al. Longterm Risk of Solid Organ De Novo Malignancies After Liver Transplantation: A French National Study on 11,226 Patients. Liver Transplant. 2018;24:1425-36. doi: 10.1002/It.25310

2. Dobrindt EM, Biebl M, Rademacher S, Denecke C, Andreou A, Raakow J, Kröll D, Öllinger R, Pratschke J, Chopra SS. De-novoUpper Gastrointestinal Tract Cancer after Liver Transplantation: A Demographic Report. Int J Organ Transplant Med. 2020;11[2]:71-80.

3. Manzia TM, Angelico R, Gazia C, Lenci I, Milana M, Ademoyero OT, et al. De novo malignancies after liver transplantation: The effect of immunosuppression-personal data and review of literature. World J Gastroenterol. 2019;25:5356-75. doi: 10.3748/wjg.v25.i35.5356.

4. Taborelli M, Piselli P, Ettorre GM, Lauro A, Galatioto L, Baccarani U, et al. Risk of virus and non-virus related malignancies following immunosuppression in a cohort of liver transplant recipients. Italy, 1985-2014. Int J Cancer. 2018;143:1588-94. doi: 10.1002/ijc.31552

5. Isik B, Yilmaz S, Kirimlioglu V, Kirimlioglu H, Yilmaz M, Sogutlu G, et al. Kaposi's sarcoma after liver transplantation from a donor with a history of ventriculoperitoneal shunt and craniotomy for primary central nervous system lymphoma: report of a case. Surg Today. 2008;38:90-4. doi: 10.1007/s00595-007-3565-x.

6. Sarac G, Ozcan KN, Baskiran A, Cenk H, Sarac M, Sener S, et al. Dermatological signs in liver transplant recipients. J Cosmet Dermatol. 2021;21. doi: 10.1111/jocd.13944.

7. Kaneko J, Sugawara Y, Tamura S, Aoki T, Sakamoto Y, Hasegawa K, et al. De novo malignancies after adult-to-adult living-donor liver transplantation with a malignancy surveillance program: Comparison with a japanese population-based study. Transplantation. 2013;95:1142-7. doi: 10.1097/TP.0b013e318288ca83.

8. Park HW, Hwang S, Ahn CS, Kim KH, Moon DB, Ha TY, et al. De novo malignancies after liver transplantation: Incidence comparison with the Korean cancer registry. Transplant Proc. 2012;44:802-5. doi: 10.1016/j.transproceed.2012.01.027.

9. Doycheva I, Amer S, Watt KD. De Novo Malignancies After Transplantation: Risk and Surveillance Strategies. Med Clin North Am. 2016;100:551-67. doi: 10.1016/j.mcna.2016.01.006.

10. Garrett GL, Blanc PD, Boscardin J, Lloyd AA, Ahmed RL, Anthony T, et al. Incidence of and risk factors for skin cancer in organ transplant recipients in the United States. JAMA Dermatology. 2017;153:296-303. doi: 10.1001/jamadermatol.2016.4920. 
11. Ducroux E, Boillot O, Ocampo MA, Decullier E, Roux A, Dumortier J, et al. Skin cancers after liver transplantation: Retrospective single-center study on 371 recipients. Transplantation. 2014;98:33540. doi: 10.1097/TP.0000000000000051.

12. Singavi AK, Harrington AM, Fenske TS. Post-transplant lymphoproliferative disorders. Cancer Treat Res. 2015;165:305-27. doi: 10.1007/978-3-319-13150-4_13.

13. Gariani K, Toso C, Philippe J, Orci LA. Effects of liver transplantation on endocrine function: a systematic review. Liver Int Off J Int Assoc Study Liver. 2016 Oct;36:1401-11. doi: 10.1111/liv.13158. Epub 2016 Jun 23.

14. Bhat M, Mara K, Dierkhising R, Watt KDS. Immunosuppression, Race, and Donor-Related Risk Factors Affect De novo Cancer Incidence Across Solid Organ Transplant Recipients. Mayo Clin Proc [Internet]. 2018;93:1236-46. doi: 10.1016/j.mayocp.2018.04.025.

15. Liu ZN, Wang WT, Yan LN. De Novo Malignancies after Liver Transplantation with 14 Cases at a Single Center. Transplant Proc. 2015;47:2483-7. doi: 10.1016/j.transproceed.2015.08.008.

16. Watt KD, Pedersen RA, Kremers WK, Heimbach JK, Charlton MR. Evolution of causes and risk factors for mortality post-liver transplant: results of the NIDDK long-term follow-up study. Am J Transplant. 2010;10:1420-7. doi: 10.1111/j.1600-6143.2010.03126.x.

\section{Tables}

Table 1. Summary of clinical characteristics of the patients with de novo malignancies. 


\begin{tabular}{|c|c|}
\hline Parameters & Total $(n=23)$ \\
\hline Age & $56.1 \pm 8.4(1-66)$ \\
\hline $\operatorname{Sex}(F / M)$ & $8 / 15(34.7 \% / 65.2 \%)$ \\
\hline \multicolumn{2}{|l|}{ Primary Liver Disease } \\
\hline$H B V$ related cirrhosis & 6 \\
\hline$H B V+H D V$ related cirrhosis & 3 \\
\hline$H B V+H C C$ & 2 \\
\hline$H B V+H D V+H C C$ & 1 \\
\hline$H C C$ & 1 \\
\hline HCV related cirrhosis & 2 \\
\hline Ethanol related cirrhosis & 2 \\
\hline Autoimmune Hepatitis & 1 \\
\hline Cryptogenic & 3 \\
\hline Biliary Atresia & 1 \\
\hline$P F I C$ & 1 \\
\hline Transplantation Type (Deceased / Living Donor) & $3 / 20$ \\
\hline Time from LT to de-novo malignancy (months) & $34.5 \pm 21.5(5-101)$ \\
\hline \multicolumn{2}{|l|}{ Types of de-novo malignancies } \\
\hline Non-melanoma skin cancer & \\
\hline $\mathrm{SCC}$ & 3 \\
\hline Malign Melanoma & 2 \\
\hline \multicolumn{2}{|l|}{ PTLD M Melanoma } \\
\hline Lung Lymphoma & 1 \\
\hline T cell Lymphoma & 1 \\
\hline Non-Hodgkin Lymphoma & 1 \\
\hline \multicolumn{2}{|l|}{ Gynecologic Cancer } \\
\hline Breast Cancer & 3 \\
\hline Over Cancer & 1 \\
\hline \multicolumn{2}{|l|}{ Myeloproliferative Disorders } \\
\hline Acute Lymphoblastic Leukemia & 1 \\
\hline Multiple Myeloma & 1 \\
\hline \multicolumn{2}{|l|}{ Colon Cancer } \\
\hline \multirow{2}{*}{\multicolumn{2}{|c|}{$\begin{array}{l}\text { Neuroendocrine tumor in small bowel } \\
\text { Renal Cell Carcinoma }\end{array}$}} \\
\hline & 1 \\
\hline \multicolumn{2}{|l|}{ Parotid Tumor } \\
\hline Nasopharynx Cancer & 1 \\
\hline \multicolumn{2}{|l|}{ Lung Cancer } \\
\hline \multicolumn{2}{|l|}{ Kaposi Carcinoma } \\
\hline Presence of Metastasis (Yes/No) & $6 / 14$ \\
\hline \multicolumn{2}{|l|}{ Treatment for de-novo malignancies } \\
\hline Surgery & 12 \\
\hline Chemotherapy & 5 \\
\hline Chemotherapy + Radiotherapy & 2 \\
\hline Non & 4 \\
\hline Current status (Alive/Ex) & $10 / 13$ \\
\hline
\end{tabular}

\section{Figures}




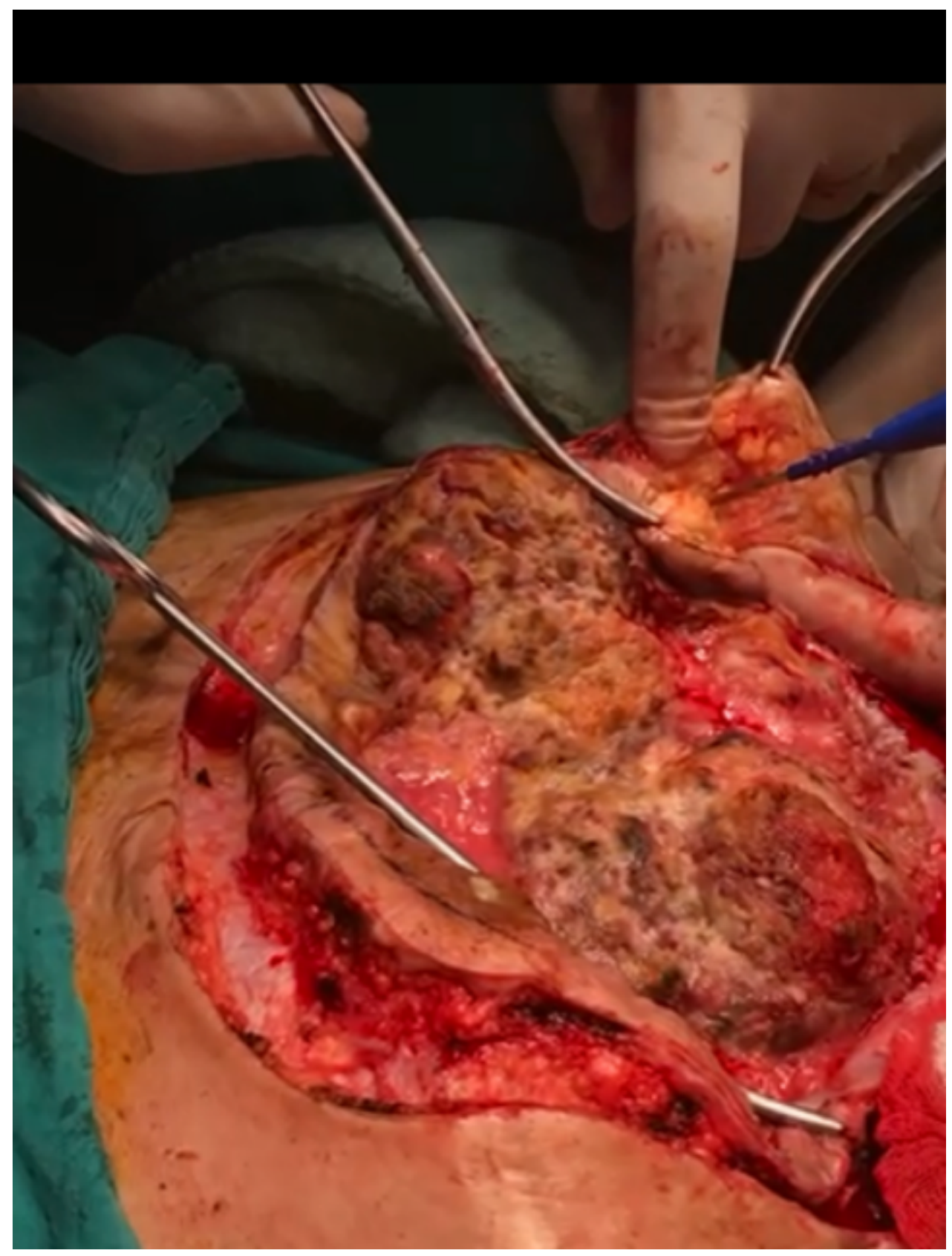

\section{Figure 1}

Intraoperative photo of aggressive squamoz cancer which was orginated from anterior abdominal wall [photo taken from S.Yilmaz archive] 

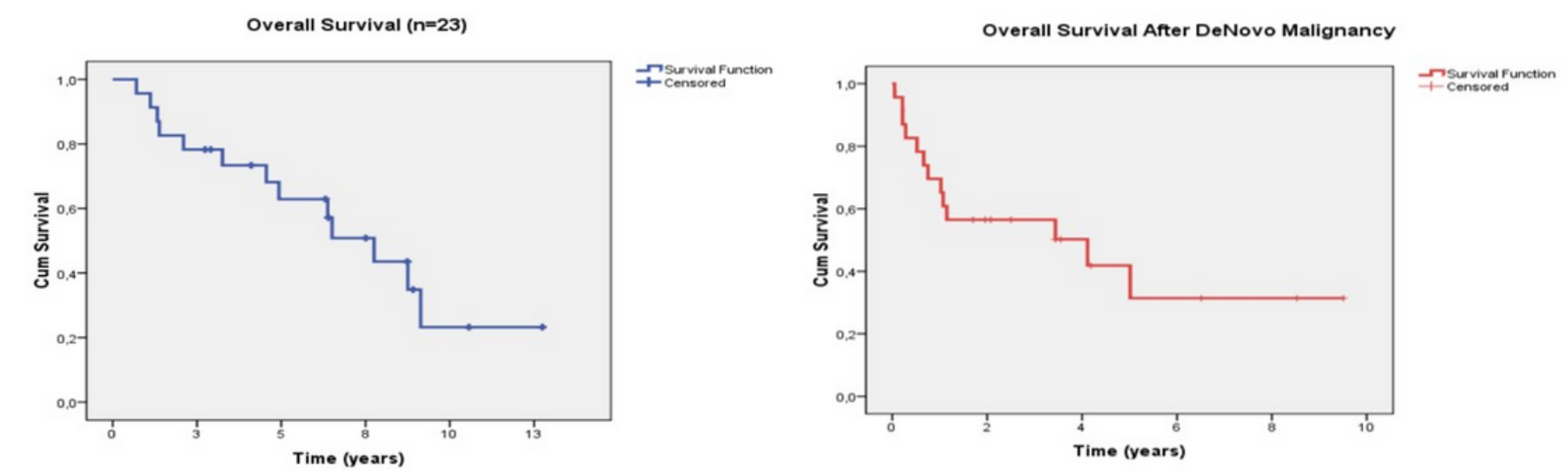

\begin{tabular}{|c|c|c|c|c|}
\hline \multicolumn{5}{|c|}{ Overall survival of the patients following liver transplantation } \\
\hline & 1-Year & 3-Year & 5-Year & Follow up (years) \\
\hline Overall survival after rate $\mathrm{LT}(\mathrm{n}=23)$ & $91.3 \%$ & $78.3 \%$ & $62.9 \%$ & $7.75 \pm 1.20(5.39-10.10)$ \\
\hline Survival after the diagnosis $(n=23)$ & $69.6 \%$ & $56.5 \%$ & $41.9 \%$ & $4.11 \pm 2.40(0.00-8.83)$ \\
\hline
\end{tabular}

\section{Figure 2}

Kaplan-Meier survival estimates of the study population showing the overall survival rate of the patients following liver transplantation $[\mathrm{A}]$ and survival rates following the diagnosis of the de novo malignancies [B]
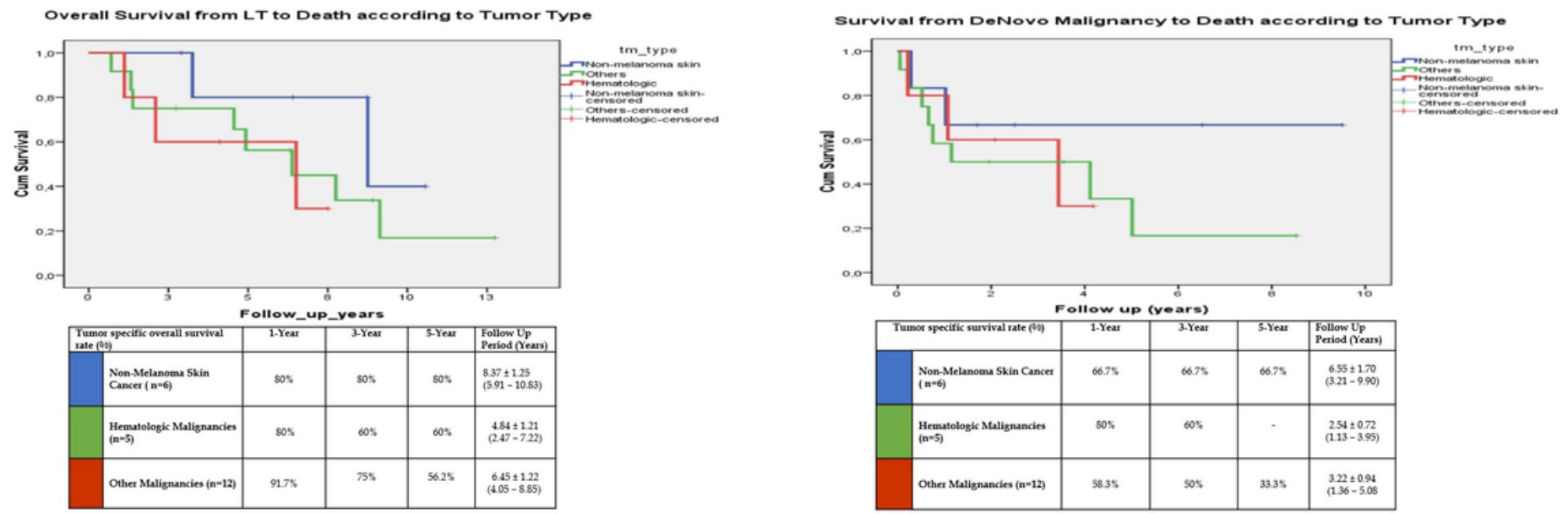

A

B

\section{Figure 3}

A: Kaplan-Meier survival estimates of the study population showing the disease-specific overall survival rate of the patients following liver transplantation. B: Kaplan-Meier survival estimates of the study population showing disease-specific survival rates following the diagnosis of the de novo malignancies. 